\title{
Estilos de aprendizaje de los estudiantes del segundo semestre de Licenciatura en matemáticas
}

\author{
Learning Styles of Second Semester Mathematics Students \\ Janz Jaramillo-Benítez ${ }^{\mathrm{a}}$, Jaime Rincón-Leal ${ }^{\mathrm{b}}$ \\ a*Estudiante del programa de Psicología. Orcid: https://orcid.org/0000-0002-5988-2699, j_jaramillo1@unisimon.edu.co, Universidad \\ Simón Bolívar, San José de Cúcuta, Colombia

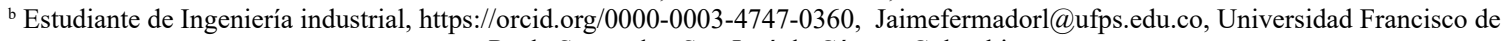 \\ Paula Santander, San José de Cúcuta, Colombia
}

Forma de citar: Jaramillo-Benítez, J. Rincón-Leal, J. Estilos de aprendizaje de los estudiantes del segundo semestre de Licenciatura en matemáticas. Eco Matemático, 10 (1), 71-75

Recibido:18 septiembre 2018

Aceptado: 15 enero 2019

\section{Palabras clave}

Estilos de aprendizaje, Pragmático, Reflexivo.

\section{Keywords}

Learning styles,

Pragmatic,

Reflective.
Resumen:La presente investigación tuvo como objetivo identificar los estilos de aprendizaje de los estudiantes de segundo semestre de Licenciatura en Matemáticas de la Universidad Francisco de Paula Santander. El estudio es cuantitativo con un diseño cuasi experimental transeccional de tipo descriptivo con una muestra de 30 estudiantes; se utilizó el cuestionario de Hábitos y Estilos de Aprendizaje (CHAEA) de Honey -Alonso. Se evidenció que el $46.67 \%$ de los estudiantes tiene predominancia en el estilo de aprendizaje reflexivo mientras que tan solo el $10 \%$ son estilo Pragmáticos, de la misma manera se utilizó el software estadístico SPSS Versión 23 para el análisis de la información.

\begin{abstract}
The present research aimed to identify the learning styles of second semester students of Mathematics at the Universidad Francisco de Paula Santander. The study is quantitative with a quasi-experimental transectional design of a descriptive type with a sample of 30 students; we used the questionnaire of Habits and Styles of Learning (CHAEA) of Honey -Alonso. It was evidenced that $46.67 \%$ of the students have predominance in the reflexive learning style while only $10 \%$ are Pragmatic style, in the same way the statistical software SPSS Version 23 was used for the analysis of the information.
\end{abstract}

*Autor para correspondencia j_jaramillo1@unisimon.edu.co 


\section{Introducción.}

En la actualidad los sectores productivos y económicos a nivel internacional y nacional están contante cambio y actualización, todo esto es posible ya que tienen como base fundamental la globalización como su punto de partida. Siguiendo por la misma línea el sistema educativo no está exento de este cambio y evolución, es por ello que el sistema educativo es un sistema abierto que debe estar en contante cabio y regulación para que no desaparezca o se destruya (Bertalanffy, 1968).

Del mismo modo, en este siglo con la nueva era digital y globalizada las cuales han llegado a tener gran impacto en la sociedad, familias y escuelas en donde se desenvuelven, así mismo, no se debe dejar de lado el contexto de cada uno de los escenarios en el que se desarrollan los individuos (Sánchez y Bello, 2016) puesto que estos son factores importantes que inciden el proceso de enseñanza y aprendizaje, es por ello que los docentes, maestros o profesionales encargados de la educación deben tener en cuenta que los individuos utilizan cada uno de sus cinco sentidos para recibir información y en su proceso de percepción e interpretación cada individuo lo realiza a partir de sus propias experiencias (Sánchez, 2015),adicional a lo anterior (Iscalá, 2017) menciona que se debe tener presente el proceso de adquisición de conocimientos, habilidades, valores $\mathrm{y}$ actitudes que tiene en estudiante a partir de su aprendizaje, de la misma manera (Santafé, 2017) que los estudiantes dependiendo de la forma en desarrollar las actividades de esa manera se da su propio aprendizaje.

Por tanto, el estudiante al hacer la interpretación de la realidad, en ese momento entra con gran relevancia el cómo hace dicho proceso cognitivo y es por ello que en cada estudiante aprende a su propio ritmo y estilo como lo afirma (Kolb, 1995) El modelo de estilos de aprendizaje presume que para aprender algo debemos trabajar o procesar la información que recibimos, desde diferentes escenarios como:1) De una experiencia directa y concreta, 2) De una experiencia abstracta, que la obtenemos cuando la leemos de algo o cuando alguien nos lo cuenta. Dichas experiencias, concretas o abstractas, se convierten en conocimiento cuando las construimos de alguna de estas dos formas: a) reflexionando y pensando sobre ellas o b) experimentando de forma activa con la información recibida.

En consecuencia, se dan 4 fases o estilos los cuales se desarrollan por toda la vida, kolb señara que el estudiante se especializa en uno o a lo mucho en dos de los siguientes estilos:

1) Activo: se involucran totalmente a experiencias nuevas, disfrutan el presente, se dejan llevar por los sucesos o acontecimientos, actúan antes de pensar en las consecuencias, se aburren en planes de largo plazo, les gusta trabajar rodeados de personas, pero siendo él el centro de las actividades, aprenden mejor en actividades cortas de resultados inmediatos, les cuesta trabajo aprender cuando tienen que ser pasivos o tiene que asimilar, analizar e interpretar datos, y cuando trabajan solos.

2) Reflexivos: son observadores y analizan las experiencias desde múltiples perspectivas, recolectan datos y los analizan de manera detallada antes de llegar a una conclusión, piensan en las consecuencias antes de actuar.

3) Teórico: adaptan e integran las observaciones con la teoría complejas y fundamentadas de manera lógica, piensan secuencialmente el paso a paso de cada acción o actividad, analizan y sintetizan la información y su sistema de valores premia la lógica y la racionalidad, no hacen juicios subjetivos, aprender mejor; a partir de modelos, teorías, sistemas con ideas y conceptos que presenten un desafío, cuando preguntan e indagan. Además, les cuesta trabajo aprender con actividades que impliquen ambigüedad e incertidumbre, situaciones emocionales y sentimentales, así cuando se trabaja sin fundamento teórico. 
4) Pragmáticos: les gusta probar las ideas, teorías y técnicas nuevas, es decir ponerlas a prueba a ver si funcionan, les gusta buscar ideas y ponerlas en prácticas inmediatamente. Son estudiantes prácticos apeados a la realidad, les gusta tomar decisiones y resolver problemas, para ellos los problemas son desafíos y siempre están en la búsqueda de la solución mas adecuada. Ellos aprenden mejor cuando: las actividades practicas se relacionan con la teoría y la practicas de manera inmediata, cuando observan al otro hacer algo, de manera contraria se les dificultad cuando: Lo que aprenden no se relaciona a una necesidad inmediata, con actividades que no tienen finalidad aparente y cuando lo que hacen no esta relacionado con la realidad.

Sin embargo, existen más modelos de estilos de aprendizajes como: a) Modelos de Felder y Silverman, b) Modelo de programación neurolingüística de Bandler y Grinder, c) Modelos de los cuadrantes cerebrales de Hermann, d) Modelos de hemisferios cerebrales, e) Modelos de las inteligencias múltiples de Gardner. A partir de todo lo anterior (Cazau, 2005) citando Revilla en donde afirma que los estilos de aprendizaje: son relativamente estables, aunque pueden cambiar es decir pueden ser diferentes en situaciones diferentes; son susceptibles de mejorarse; y cuando a las personas se para comunicar se utiliza la estrategia según su propio estilo de aprendizaje, aprenden con más efectividad.

Sin embargo, se debe tener en cuenta que la elaboración personal que resulta de la reflexión en que el individuo explora, utiliza y adecua las posibilidades de sus procesos cognoscitivos y que, en unión su experiencia personal, favorece de forma autorregulada la asimilación de los contenidos necesarios para su vida y su aprendizaje (Mesa, 2006).

En este sentido, los docentes en los niveles de primaria, secundaria y universitaria tienen dificultades orientar y comunicar la información pertinente para a su vez convertirla en conocimiento adecuado a sus estudiantes en cualquiera de sus niveles. Lo anterior se debe a deficiencias entre el sujeto emisor (docente) y el individuo receptor(estudiante) es decir de la manera en que el docente cómo enseña en contraste del qué aprende y lo que debe ser aprendido por el estudiante.

Por lo tanto, la estrategia que se debe utilizar al inicio de todo proceso de aprendizaje, es el de identificar los canales y estilos de aprendizaje de los estudiantes, con el fin de utilizar la metodología adecuada para que el proceso de enseñanza aprendizaje sea el esperado.

\section{Materiales y Métodos}

La investigación sigue un el paradigma Positivista el cual se califica en cuantitativo, empírico-analítico, racionalista, sistemático gerencial y científico tecnológico (Ricoy, 2006) con un enfoque cuantitativo como lo define (Hernadez Sampieri, Fernández Collado, \& Baptista Lucio, 2014) que por la naturaleza de la investigación los datos recolectados se pueden analizar y con las mediciones obtenidas pueden utilizando métodos estadísticos, y se extrae una serie de conclusiones, del mismo modo el diseño de la presente investigación es no experimental, transaccional, puesto que no existe manipulación de ninguna de las variables además las variables se analizan de la forma espontánea y natural, tal como se presentan los hechos y solo aplicamos en un solo momento para aplicar el cuestionario y la recolección de información. (Sampieri, 2018).De la misma manera el alcance de la investigación descriptivo ya que consiste en describir los estilos de aprendizaje de los estudiantes del programa Académico de Licenciatura en Matemáticas.

\section{Instrumento}

Se aplicó el cuestionario de Hábitos y Estilos de Aprendizaje (CHAEA) de Honey y Alonso el cual 
describe cuatro estilos o preferencias específicas a la hora de abordar el proceso de aprendizaje: a. Estilo Activo: Implicación activa y sin prejuicios en nuevas experiencias. b. Estilo Reflexivo: Observación de las experiencias desde diversas perspectivas. Prioridad de la reflexión sobre la acción. c. Estilo Teórico: Enfoque lógico de los problemas. Integración de la experiencia dentro de teorías complejas. Y d. Estilo Pragmático: Experimentación y aplicación de las ideas, el cuestionario este compuesto por 80 ítems distribuidos de manera estratégica y distribuido en 4 grupos según los estilos (Alonso \& Honey, 1994), para el análisis estadístico se utilizó el software SPSS versión 23.

\section{Resultados y discusión}

La población estuvo conformada por 240 estudiantes en edades entre 17 y 31 años quienes actualmente son estudiantes de Licenciatura en Matemáticas en la Universidad Francisco de Paula Santander, y la muestra se estuvo constituida por 30 estudiantes que pertenecían al segundo semestre, entre los cuales el $57 \%$ equivalentes a 17 estudiantes encuestados son de sexo masculino, mientras $43 \%$ equivalentes a 13 estudiantes de sexo femenino, seguidamente el 50\% son de estrato $1 \mathrm{y}$ el otro $50 \%$ de estrato 2 equivalente a 15 estudiantes, la Lateralidad de los estudiantes es el $6.7 \%$ son ambidiestros, el 10\% izquierdos y el $83.3 \%$ son de lateralidad derecha. Mientras el 17\% se graduó como bachiller de una Institución privada mientras que $83 \%$ de una institución pública, a mismo el $3.3 \%$ son casados, el 6,7\% están en unión libre y el $90 \%$ son solteros.

El Cuestionario de Honey-Alonso sobre estilos de aprendizaje, tuvo como propósito identificar los cuatro estilos de cada uno de los participantes a la hora de abordar los procesos cognoscitivos del aprendizaje: El estudiante al responder con un sí equivale a 1 y No es igual a 0 . Luego se suman cada uno de los ítems y se identifica 1 o 2 estilos predominantes en cada uno de los participantes.
En la figura 1 se encuentran relacionados los estilos de aprendizaje de los estudiantes participantes del cuestionario, el 20\% equivalente a(6) estudiantes, son de estilo activo de los cuales el 33\% mujeres y el $67 \%$ hombres. El 10\% equivalente a (3) estudiantes, son de estilo pragmático con $67 \%$ mujeres y $33 \%$ hombres. El 46.67\% equivalente a (14) estudiantes, son de estilo reflexivo distribuidos en $43 \%$ mujeres y $57 \%$ hombres y finalmente, el $23.33 \%$ equivalente a (7) personas, son de estilo teórico, en donde el $43 \%$ mujeres y $57 \%$ hombres. A partir de los resultados obtenidos en la figura se puede afirmar que el estilo de aprendizaje dominante en los estudiantes del segundo semestre de Licenciatura en Matemáticas es el estilo reflexivo, así como se observa que el estilo con menor dominancia es el pragmático.

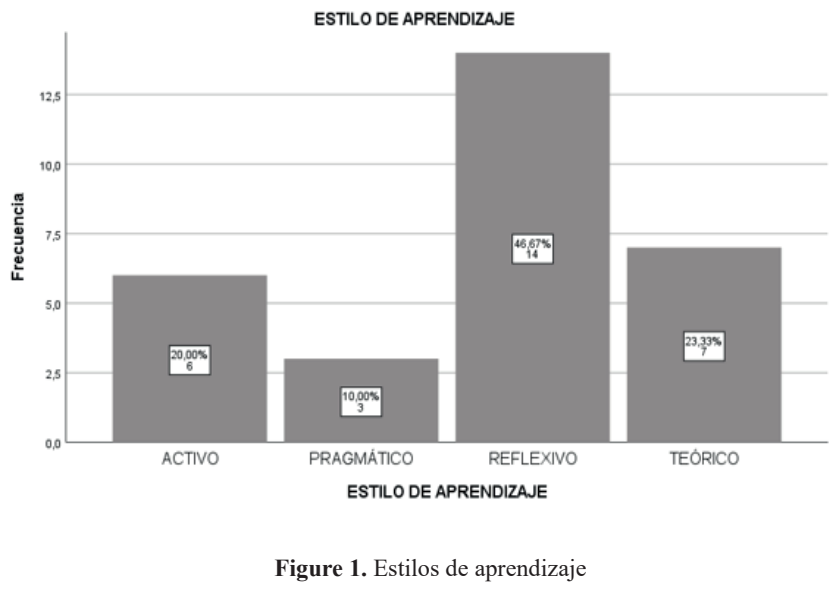

\section{Conclusiones}

Se puede afirmar que, al identificar los diferentes estilos de aprendizaje de cada uno de los estudiantes durante su proceso de formación, el más significativo es el estilo reflexivo, el cual tiene relación con el programa académico de licenciatura en Matemáticas los cuales son analíticos, observadores, recopiladores, detallistas, argumentador, previsor de alternativas, observador de conductas y comportamientos, piensan antes de actuar todo esto se relaciona al momento de orientar una clase, de planificar diarios programadores, de tener dominio de grupo, de utilizar diversas estrategias y metodologías para mejorar el proceso de enseñanza y aprendizaje en las instituciones 
educativas. Mientras que el estilo de aprendizaje pragmático fue el más bajo entre los participantes, los cuales son experimentadores, prácticos, realistas, concretos y claros.

\section{Referencias}

Alonso, C. M., \& Honey, P. (1994). Cuestionari de Hábitos y Estilos de Aprendizaje(CHAEA). Reino Unido.

Bertalanffy, L. V. (1968). Tería General De los Sistemas. Mexico D.F: Fondo de cultura Económica.

Cazau, P. (2005). Estilos de aprendizaje:Generalidades. 25.

Hernadez Sampieri, R., Fernández Collado, C., \& Baptista Lucio, p. (2014). Definiciones de los enfoques cuantitativos, sus similitudes y diferencias. En Metodologia de la investigación sexta edicion (págs. 2-21). Mexico : McGraw Hill Education.

Iscalá, D. (2017). Fortalecimineto del pensamiento numèrico a trave's de estrategias didácticas que desarrollen competencias comunicativas en los estudiantes del grado tercero de esducación primaria. Eco maremático 8(1), 49-61.

Kolb, D. (1995). Estilos de aprendizaje. Sloan School of Management.

Mesa, A. S. (2006). Prevencion de la diabetes y estilos de aprendizaje. Interpsiquis VII.

Ricoy, C. (2006). Contribución sobre los paradigmas de investigación. Revista do Centro de Educación, 11-22.

Sampieri, R. (2018). Metodología de la investigación: las rutas cuantitativa, cualitativa y mixta. Mexico: McGraw Hill.

Sánchez Teruel, D., \& Robles Bello, M. A. (2016). Riesgos y potencialidades de la era digital para la infancia y la adolescencia. Dialnet, 19.

Sánchez, J. J. (2015). Percepción y Experiencia. EPISTEME, 21-36.

Santafé, Y. (2017). Fortalecimineto de competencias cientìicas en la asignatura de física para estudiantes de undécimo grado en Colombia. Eco matemático 8(1), 34-42. 